\title{
Hydrodynamics During the Transient Evolution of Open Jet Flows from/to Wall Attached Jets
}

\author{
A. Valera-Medina ${ }^{1} \cdot$ H. Baej ${ }^{1}$
}

Received: 15 September 2014 / Accepted: 8 February 2016 / Published online: 4 March 2016

(C) The Author(s) 2016. This article is published with open access at Springerlink.com

\begin{abstract}
Swirl stabilized flows are the most widely deployed technology used to stabilize gas turbine combustion systems. However, there are some coherent structures that appear in these flows close to the nozzle whose occurrence and stability are still poorly understood during transition. The external recirculation zone and the Precessing Vortex Core to/from the Coanda effect are some of them. Thus, in this paper the transition of an Open Jet FlowMedium Swirl flow pattern to/from a Coanda jet flow is studied using various geometries at a fixed Swirl number. Phase Locked Stereo Particle Image Velocimetry and High Speed Photography experiments were conducted to determine fundamental characteristics of the phenomenon. It was observed that the coherent structures in the field experience a complete annihilation during transition, with no dependency between the structures formed in each of the flow states. Moreover, transition occurs at a particular normalized step size whilst some acoustic shifts in the frequencies of the system were noticed, a phenomenon related to the strength of the vortical structures and vortices convection. It is concluded that a transient, precessing, Coanda Vortex Breakdown is formed, changing flow dynamics. The structure progresses to a less coherent Trapped Vortex between the two states. During the phenomenon there are different interactions between structures such as the Central Recirculation Zone, the High Momentum Flow Region and the Precessing Vortex Core that were also documented.
\end{abstract}

Keywords Swirling flows · Coherent structures · Coanda $\cdot$ Precessing vortex core Trapped vortex

A. Valera-Medina

valeramedinaa1@ cardiff.ac.uk

1 School of Engineering, Cardiff University, Queen's Building, the Parade, Cardiff, Wales, UK 


\section{Nomenclature}

$\begin{array}{ll}\text { CoJF } & \text { Coanda Jet Flow } \\ \text { COVB } & \text { Coanda Vortex Breakdown to Radial Jet Flow } \\ \text { CRZ } & \text { Central Recirculation Zone (coherent structure) } \\ \text { ERZ } & \text { External Recirculation Zone } \\ \text { D } & \text { Nozzle diameter [m] } \\ \text { HMFR } & \text { High Momentum Flow Region } \\ \text { OJF-MS } & \text { Open Jet Flow-Medium Swirl } \\ \text { PIV } & \text { Particle Image Velocimetry } \\ \text { PVC } & \text { Precessing Vortex Core } \\ \text { St } & \text { Strouhal number [-] } \\ \text { TV } & \text { Trapped Vortex } \\ \mathrm{U} & \text { Axial velocity [m/s] } \\ \text { V } & \text { Radial velocity [m/s] } \\ \mathrm{W} & \text { Tangential velocity [m/s] } \\ \alpha & \text { Nozzle angle }\left[{ }^{\circ}\right] \\ |\Delta \mathrm{X}| & \text { Absolute step size }[\mathrm{m}] \\ \lambda_{c i} & \text { Imaginary part of the complex eigenvalue of the velocity gradient tensor }\end{array}$

\section{Introduction}

Future flexible power plants will require new and improved systems for flexible usage of a variety of alternative fuels. However, the use of such fuels requires that fundamental flow structures close to the burners are well understood, especially for multi-burner applications or flames that are not highly confined. There are well known structures that are essential to the stability of current burners, i.e. Central Recirculation Zone, Precessing Vortex Core, etc. [1]. However, there are some structures whose interaction with the localised flow field during transition are still poorly understood.

One of these structures is the External Recirculation Zone (ERZ) observed in Open Jet Flow-Medium Swirl (OJF-MS) flames with swirl numbers close to 1.0. Previous studies on swirling flows $[2,3]$ have identified the importance of such a region as a mechanism of stability to anchor flames and recirculate hot products, complementing the action of the Central Recirculation Zone (CRZ), a coherent structure that appears in the vicinity of the shearing flow and inside of the flame in OJF-MS. The CRZ is a product of the decaying pressure and the swirling movement of the flow $[4,5]$. Inevitably, the structure can produce complex oscillations as high vorticity interacts with other structures whilst varied entrainment rates can encourage equivalence ratio and heat release variations [6]. The OJF-MS features are similar to those of the Open Jet Flow-High Swirl, Fig. 1, but with less concave patterns due to longer and weaker CRZs product of increased axial velocities.

Another important type of exhaust flow with swirl is the Coanda Jet Flow (CoJF), which contrary to the OJF-MS pattern occurs when ejected gases from a burner attach to the flat mounting plate on which the nozzle exit is connected $[7,11]$. When a jet of fluid is passed over a curved or a strongly divergent nozzle the jet is pulled onto the curved surface as a result of the low pressure region which develops as entrainment by the jet removes gases from the region between the jet and the surface. The balance between the inward radial pressure gradient and the outward centrifugal force then holds the jet to the wall [8]. The 
a

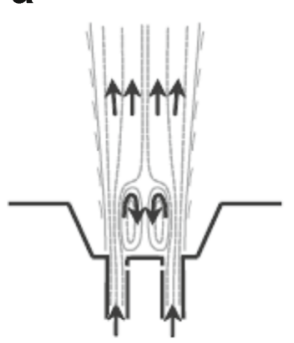

b

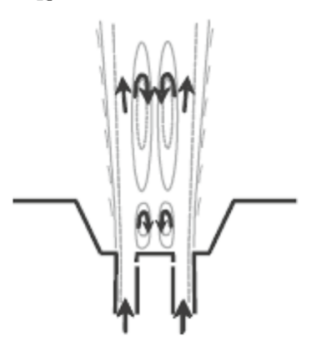

C

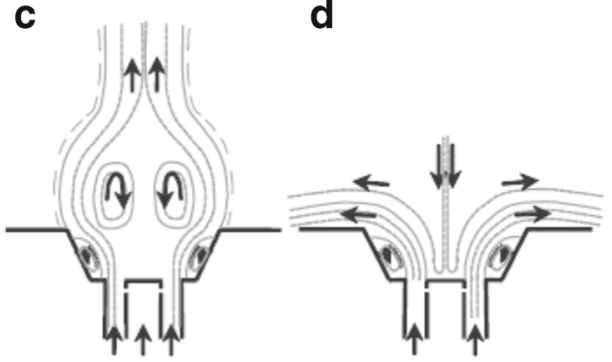

Fig. 1 Schematic view of the different flow patterns. a) Closed Jet Flow; b) Open Jet Flow Low Swirl; c) Open Jet Flow High Swirl (OJF-HS); d) Coanda Jet Flow (CoJF) [7]

substantial flow deflection offered by the Coanda principle is generally accompanied by enhanced levels of turbulence. A related, although undesirable side effect is the significant increase in associated noise levels $[8,12]$. Applications of the phenomenon vary from aerospace design stealth technology to burners and medicine applications $[8,13,14]$. However, the complex nature of the flow in 3 dimensions is still a challenge and many of its effects are still barely understood [15].

Other important structures appear in the system as a consequence of the swirling motion of the flow. The Precessing Vortex Core (PVC) is another one of them. Syred and Beer [2] showed that this time dependent, hydrodynamic instability could be characterised by a non-dimensional frequency parameter (a form of Strouhal number which correlates eddy shedding frequencies to Reynolds number), momentum and fluctuating pressure. Although some systems have been classified as precessing or stable swirling flows [1], the mechanism of manifestation of the PVC event is still unclear. Some authors have studied the phenomena under isothermal conditions [16], giving ideas about the movement of the core as a manifestation of the compressing-expansive mechanism in inner parts of the structure. A modern idea stipulates that its formation comes from a series of small eddies generated by the CRZ [4]. However, the transition of different flow patterns could give a better understanding of its appearance and behaviour during this process. Thus, the transition of the ERZ to/from the Coanda effect with swirl burners could be a phenomenon that unlocks some interesting features of this and other structures, a process that still requires extensive characterization.

Vanoverberghe [10] studied the phenomena via the transition of different flame regimes in a generic swirl burner at different swirl numbers, observing that cold flows and Swirl numbers were to a large degree responsible for the type of flame pattern with combustion superimposed on it. Vanierschot [7] showed that as a consequence of the increase in the deflection angle of the exhaust flow when decreasing the swirl the flow near the outlet wall of the exhaust nozzle sticks and increases the velocity. This causes the local pressure to decrease, deflecting the flow and producing the Coanda effect. However, effects such as ERZ, CRZ and PVC formation and interaction with the Coanda flow have yet to be fully investigated.

Modellers have tried to predict the Coanda effect and the ERZ region for many applications. Mirkov and Rasuo [17] using in-house software and the k- $\omega$ Shear Stress Transport model accomplished the analysis of an Unmanned Aerial Vehicle using the Coanda effect, denoting the high attachment of the upper flow and its separation at the bottom of the geometrical array, with the appearance of vortical structures similar to the ones observed with 
bluff bodies. Rumsey and Nishino [18] showed that with flows under 0.64 Mach number the solutions between incompressible and compressible RANS were very similar when analysing the Coanda effect on airfoils. Drangan and Stanciu [19] used the k- $\varepsilon$ model to observe a new super circulation technique to reduce drag and improve lift in aerospace applications. Tavakoli and Hosseini [20] investigated the relation between the Swirl number and the flow pattern using different angles in an axial vaned swirler used for ventilation applications. Although these and more advanced techniques such as LES [21, 22] have been used for the study of the Coanda effect, the computational results have not shown the transient nature of the process. The transition from a vortex breakdown dominated flow to a Coanda jet has also been studied by Singh and Ramamurthi [21], who showed that the increase in swirl in sharp-edged nozzles produce a system dominated by centrifugal forces that spread the flow in the radial direction, producing the particular shape of the Coanda effect. Their results showed the appearance of vortical structures that migrated through the system. However, the computational results did not show a close correlation to the experimental values obtained by the group.

For the External Recirculation Zone and PVC, extensive literature has shown their appearance just downstream the burner nozzle in power and propulsion applications [1, 23, 29], but there is limited information about the transition of these structures. Mullyadzhanov et al. [30] presents a coherent literature review of the topic with LES results showing the hysteresis of the transition phenomenon with some conclusions on the differences between hot and cold regimes. Vanierschot and Van den Bulck [31,32] describe different flow patterns produced by these configurations emphasizing some of the features during the transition process. They focus on the hysteresis of the phenomenon. However, other coherent structures apart from recirculation zones are briefly discussed in these studies. Thus, more work is required to quantify this process.

Thus, hydrodynamic fundamentals and coherent structures during the evolution to/from a Coanda Jet with a medium swirl device which has already passed through a vortex breakdown has been briefly investigated. Therefore, swirling flows using a generic burner are studied in the present work via experimental laser and visualization techniques. The aim of the study is to observe how the transition from/to a CoJF to/from a conical OJF-MS pattern takes place with different geometries, giving insights of the evolution of different structures across the flow field.

\section{Setup}

A swirl burner constructed from stainless steel was used to examine the transition behaviour under atmospheric conditions (1bar, 293K) at Cardiff University. A diagram of the generic burner is presented in Fig. 2 and detailed somewhere else [33]. A single tangential inlet (a) feeds the premixed air and fuel to an outer plenum chamber (b) which uniformly distributes the gas to the slot type radial tangential inlets (c). Swirling premixed air and fuel then passes into the swirl chamber (d), then into the exit nozzle (e). The central diffusion fuel injector (f) extends centrally through the combustor body to the exhaust.

The system was fed using compressed air through flexible hoses and a Coriolis meter for flow rate control. Methane from cylinders was used as fuel, fed through flexible hoses passing through another Coriolis meter. All the cases were analysed from $\operatorname{Re} \sim 9,000$ to 27,000 , thus providing details of the jets in fully turbulent regimes for swirling flow at $S=$ 1.05 [1]. Equivalence ratios ranged from 0.58 to 1.02 . 


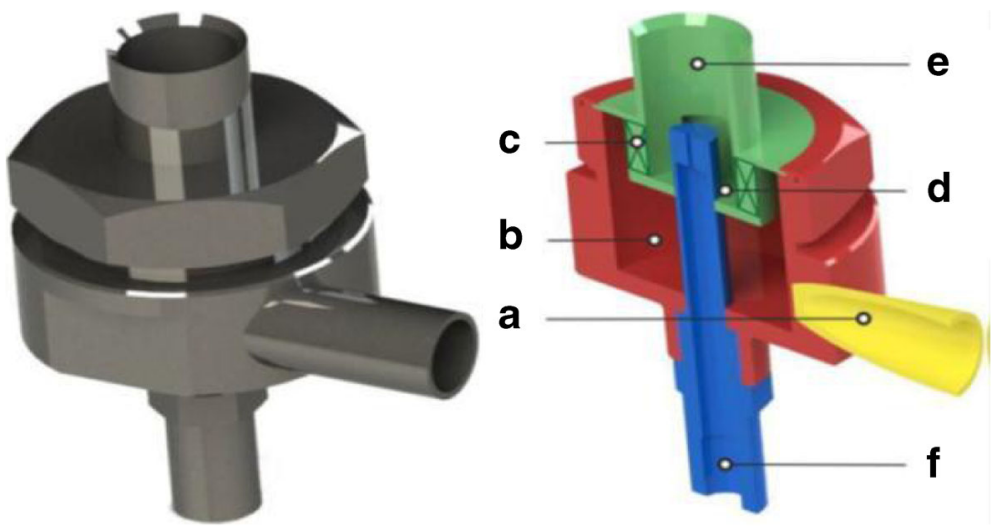

Fig. 2 Burner (left) and schematic of the components of the burner (right)

Strong PVCs and associated structures were found. Their frequencies were characterised via a PCB Piezotronics 378B02 condenser microphone located $0.01 \mathrm{~m}$ upstream from the burner outlet which tracked the frequency changes of the High Momentum Flow Region (HMFR) $[34,35]$ formed by the precessing shearing flow. The microphone condenser signal was redirected to a signal conditioner with low and high band pass to recognise frequencies above $10 \mathrm{~Hz}$ and up to $2,500 \mathrm{~Hz}$. The reconditioned signals were redirected to trigger a BNC Model 500 Pulse Generator, whose TTL signal was sent to a Dantec Stereo PIV system for triggering purposes. The latter consists of a dual cavity Nd: YAG Litron Laser of 532 $\mathrm{nm}$ capable of operating at $15 \mathrm{~Hz}$. Dantec Dynamics laser sheet optics (9080X0651) were used to convert the laser beam into a $1 \mathrm{~mm}$ thick sheet. To record the images a pair of $\mathrm{Hi}$ Sense MkII Cameras model C8484-52-05CP were used, with 1.3 MPixel resolution at 8 bits. $60 \mathrm{~mm}$ Nikon lenses were used for resolution purposes, which allowed a field of view of approximately $75 \times 75 \mathrm{~mm}$, with a resolution of 5.35 pixels per mm and a depth of view of $1.5 \mathrm{~mm}$. PIV measurements were obtained a couple of $\mathrm{mm}$ from the tip of the nozzle to avoid laser reflection. The inlet air was seeded with $\mathrm{Al}_{2} \mathrm{O}_{3}$ for the cases with combustion by a compressed air fed accumulator positioned $2 \mathrm{~m}$ upstream of the burner inlets. A water nebulizer was used under isothermal conditions to observe the flow patterns. The entire system was triggered at $90 \%$ of the highest peak observed after 5 minutes of free running, a technique previously demonstrated [35]. The repetition rate was set at $1 / 100$ of the HMFR frequency, which was measured via a 4 channel, $2 \mathrm{G} / \mathrm{s}$ Tektronix Oscilloscope.

After acquisition of the Stereo PIV data, a frame-to-frame adaptive correlation technique was then carried out with a minimum interrogation area of $32 \times 32$ pixels and a maximum of $64 \times 64.300$ pairs of frames were used to create an average phase locked velocity map after tests showed 500 pair of frames gave similar results in an independency test campaign. PIV correlation adaptivity allowed the visualization of coherent structures, with a spatial smoothing of $\sim 15 \%$ of the entire field of view. The accuracy of the results relies on the careful focusing of the image, correct injection of particles and masking of regions with very low flow. Axial-radial velocity maps were created over the ranges of -5.00 to $8.00 \mathrm{~m} / \mathrm{s}$ for $\mathrm{U}$ and 0.00 to $7.00 \mathrm{~m} / \mathrm{s}$ for total velocity for the OJF-MS and CoJF, respectively. Radialtangential maps were created over the range of -10.00 to $20.00 \mathrm{~m} / \mathrm{s}$ for total velocity, with $\sim 90 \%$ of vectors within these velocity ranges. In order to reduce the parallax error, focusing 


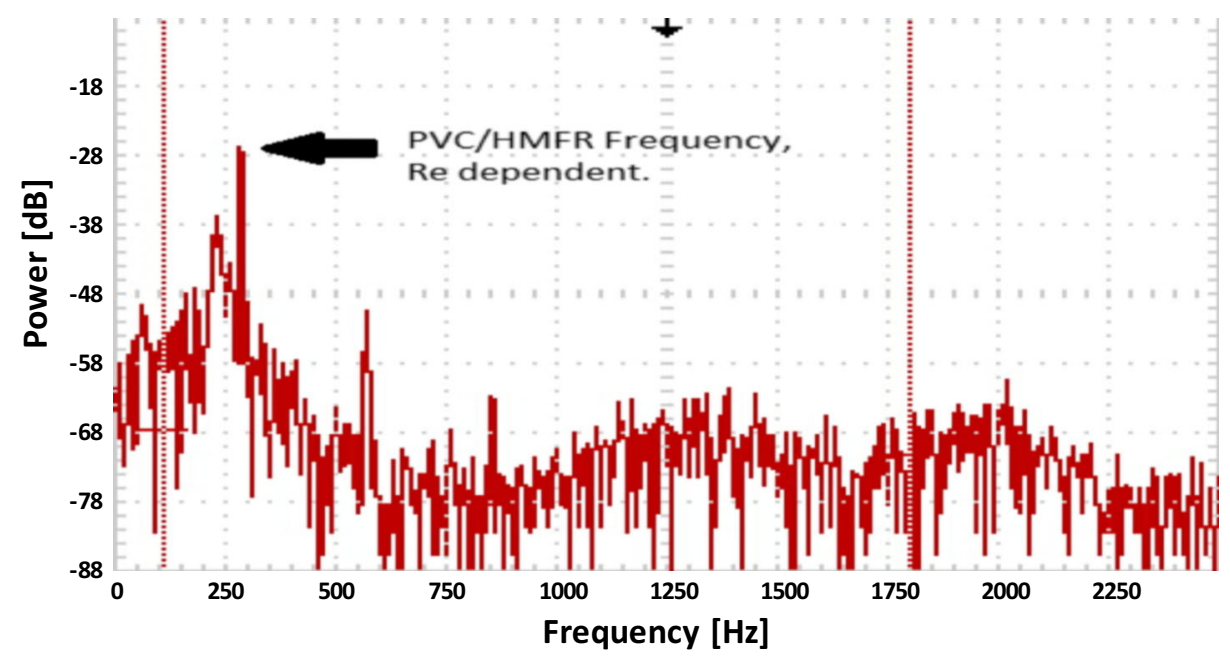

Fig. 3 Fast Fourier Tranform frequency analysis at $\sim 10,100$ Re. PVC/HMFR peak depends on Re

was achieved using the corners of a grid and correcting the position of the lens (Scheimflug correction). The line of view of the camera was positioned exactly in the middle of the nozzle using a grid provided by the laser manufacturer. Finally, the grid was used to correct via software any remaining positioning issue.

The first frequency mode observed was the Helmholtz resonance of the system at 200 $\mathrm{Hz}$, being always the same at all flowrates. The second frequency mode found was that of the PVC/HMFR. The frequency of the PVC/HMFR was observed to be around $240-750 \mathrm{~Hz}$, depending on Re, Fig. 3. Experiments were repeated 10 times. Discrepancies in results $(\sim 5 \%)$ were caused by fluctuations in the compressed air supply.

A Fastcam APX RS high speed camera operating at 1000 frames/s was also used with a $105 \mathrm{~mm}, 1: 2.8$ Nikon lens to characterize the transition. The resulting images were analysed using the PFV ver 2.4.1.1 software. Different geometries were used to better understand the phenomenon. Different normalized step sizes, $|\Delta \mathrm{X}| / \mathrm{D}$, were used from 0.000 to 0.145, Fig. 4. Nozzle angles, $\alpha$, of $30^{\circ}, 45^{\circ}, 60^{\circ}$ and $90^{\circ}$ were also utilized. Outlet diameter and Swirl number were kept constant at $0.0280 \mathrm{~m}$ and 1.05 , respectively. Geometrical changes were based on the observations of Sidhu et al [11] who used a radiused exhaust nozzles leading to a flat exhaust plate on the exhaust of a vortex diode. Thus, it was expected that the aerodynamics would change considerably as the step between the flat exhaust plate and the nozzle lip was altered.

\section{Results and Discussion}

This section links experimental results to demonstrate the transition of the flow from/to a Conical Jet Flow to/from an OJF-MS. Results show that the flow can fall under 3 different conditions, A) Coanda stabilization with the appearance of a Coanda Vortex Breakdown; B) Transition, where a Trapped Vortex (TV) leads to the detachment of the flame and destruction of flow patterns; C) Open Jet stabilization with the formation of the CRZ and the appearance of the PVC, ERZ and HMFR. 
Fig. 4 Geometrical changes at the exit nozzle. Step size $(\Delta \mathrm{X})$ and nozzle angle $(\alpha)$

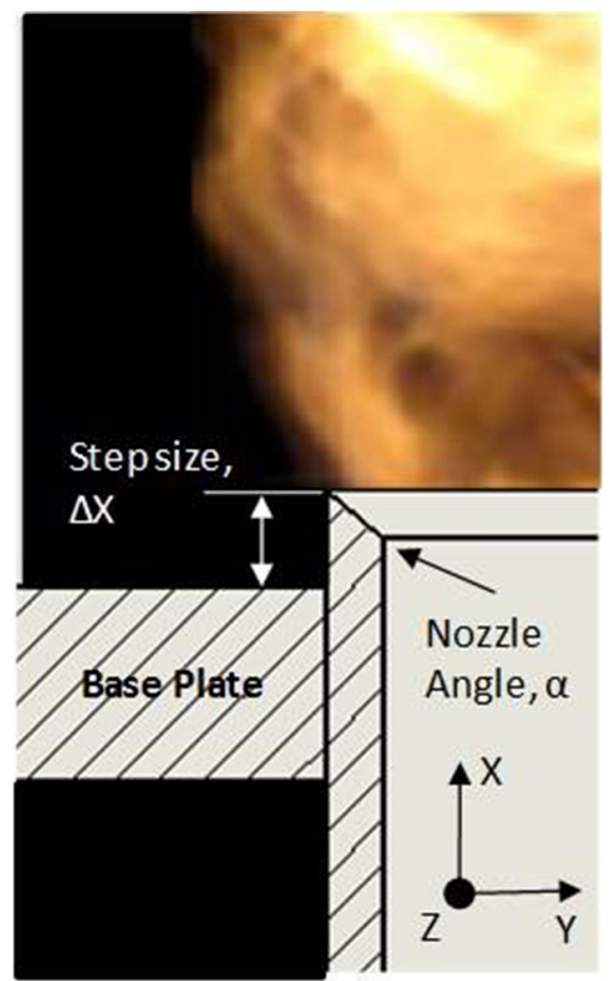

\subsection{Combustion experiments}

Experiments with combustion were run using $\alpha=45^{\circ}$. Careful ignition of the flow was attempted, as it was observed that a sudden uncontrolled reaction would lift the flow not allowing the visualization of the Coanda effect. It was observed that the flame behaved similarly to other OJF-MS conical flames at $|\Delta X| / D=0.145$ and 0.107 . This was denoted by a Rankine tangential velocity profiles, a CRZ and HMFR characteristic of such flows, Fig. 5. Thereafter, the base plate was moved towards the nozzle and positioned at $|\Delta \mathrm{X}| / \mathrm{D}=0.072$. This change in geometry produced a CoJF. $|\Delta X| / D$ was then changed to 0.036 and 0.000 , and the same flat distribution was observed, Fig. 6. In the CoJF the flat profile allows greater quantities of air entrainment, whilst producing series of vortical structures between the incoming air and the flow attached to the base plate $[11,21]$.

A closer look at the stereo PIV results and visual inspection showed how a trapped vortex interacts with the flame, Figs. 7 and 8 . The high velocity exhaust flow bends towards the exhaust plate trapping the vortex. This can be observed at $0.193 \mathrm{D}$ from the outlet, Fig. 7c, where a positive peak appears in the axial velocity component $U$. The vortex seems to be strong only on one side of the system. Results also show that the tangential velocity component $\mathrm{W}$ rapidly becomes irregular and intermittent as the distance from the centre line becomes greater than the external diameter. Figure 8 shows the main vortex at the lefthand side through both a vorticity and swirling strength analyses. Unfortunately, vorticity not only identifies vortex cores but also shearing motion, thus requiring another manner to extract vortical cores. An employed method is to define the Swirling strength of the flow. 


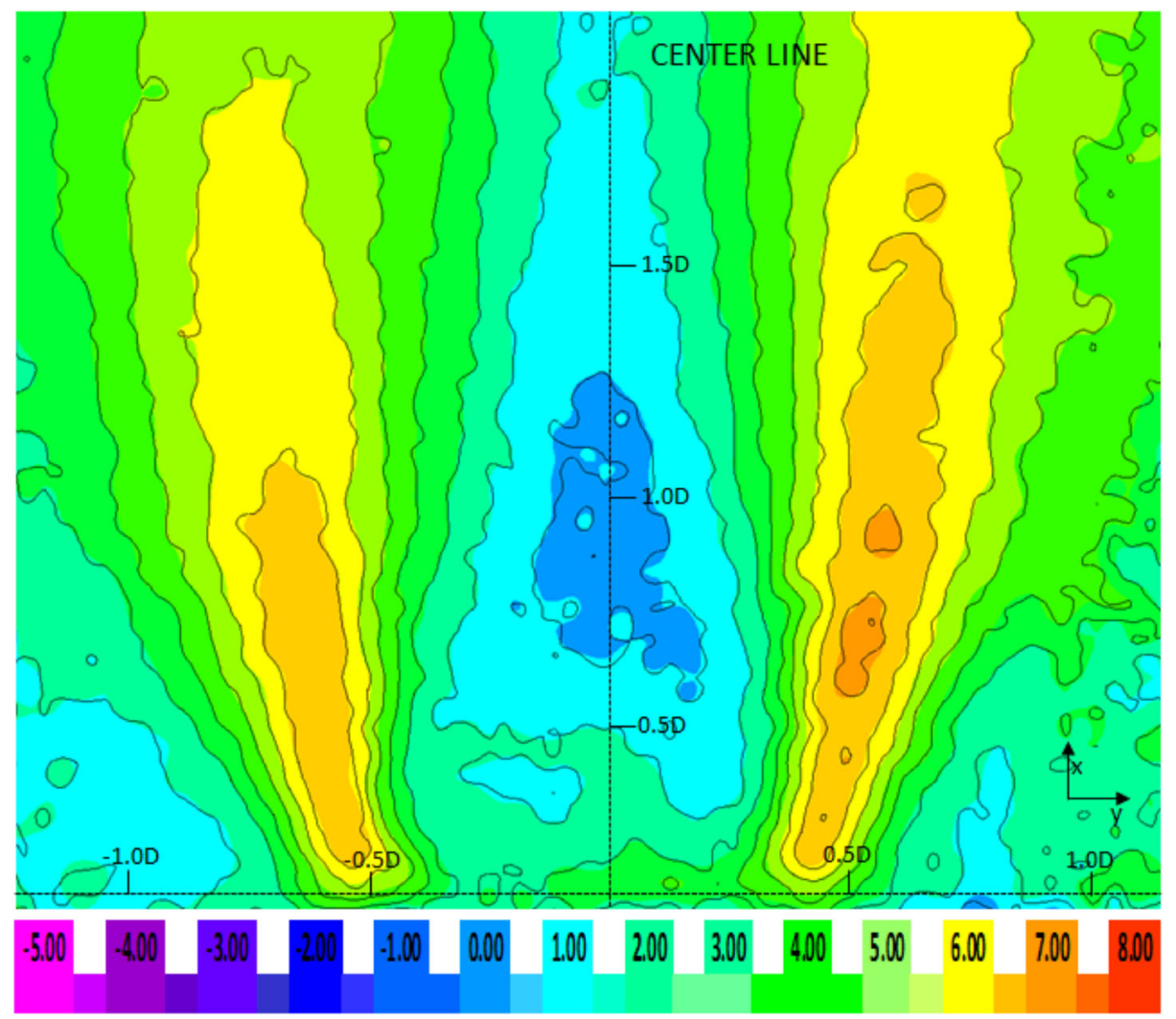

Fig. 5 OJF-MS shape. $\operatorname{Re}^{\sim} 15,500,|\Delta X| / D=0.145$, equivalence ratio $0.58, \alpha=45^{\circ}$. $U$ velocity in $[\mathrm{m} / \mathrm{s}]$

The technique uses local velocity gradient tensors which have a real eigenvalue and might have a pair of complex conjugate eigenvalues. The latter can be related to shorter, circular ellipses (i.e. eddies) [36, 37]. Thus, swirling strength is defined as the imaginary part of the complex eigenvalue of the velocity gradient tensor. The square of the imaginary part, or value returned as Swirling strength, can be obtained as [38],

$$
\lambda_{c i}^{2}=\frac{1}{4}\left(\frac{\partial U}{\partial x}\right)^{2}+\frac{1}{4}\left(\frac{\partial V}{\partial y}\right)^{2}-\frac{1}{2} \frac{\partial U}{\partial x} \frac{\partial V}{\partial y}+\frac{\partial V}{\partial x} \frac{\partial U}{\partial y}
$$

Thus, the corresponding eigenvalues will only be complex if this number is negative. Local minima of negative-valued swirling strength can be used to identify vortex cores, while positive values indicates areas of the flow where shear may be present without swirling motion. Therefore, Swirling strength results, Fig. $8 \mathrm{~B}$, confirm the existence of a vortex that due to its strength creates a counter-rotating zone with shearing flow above it. It is clear that the shear produced close to the vortex will considerably affect local equivalence ratios.

Transition between the $\mathrm{CoJ}$ and OJF-MS was observed in the range between $|\Delta \mathrm{X}| / \mathrm{D}=0.072$ and 0.089 . Experiments in this range were conducted with increments of $\Delta(|\Delta \mathrm{X}|) / \mathrm{D}=0.004$ to correctly determine the crucial position for transition. It was observed that the jet had a comparatively randomised pattern at $|\Delta X| / D=0.082$. The experiments were run at 


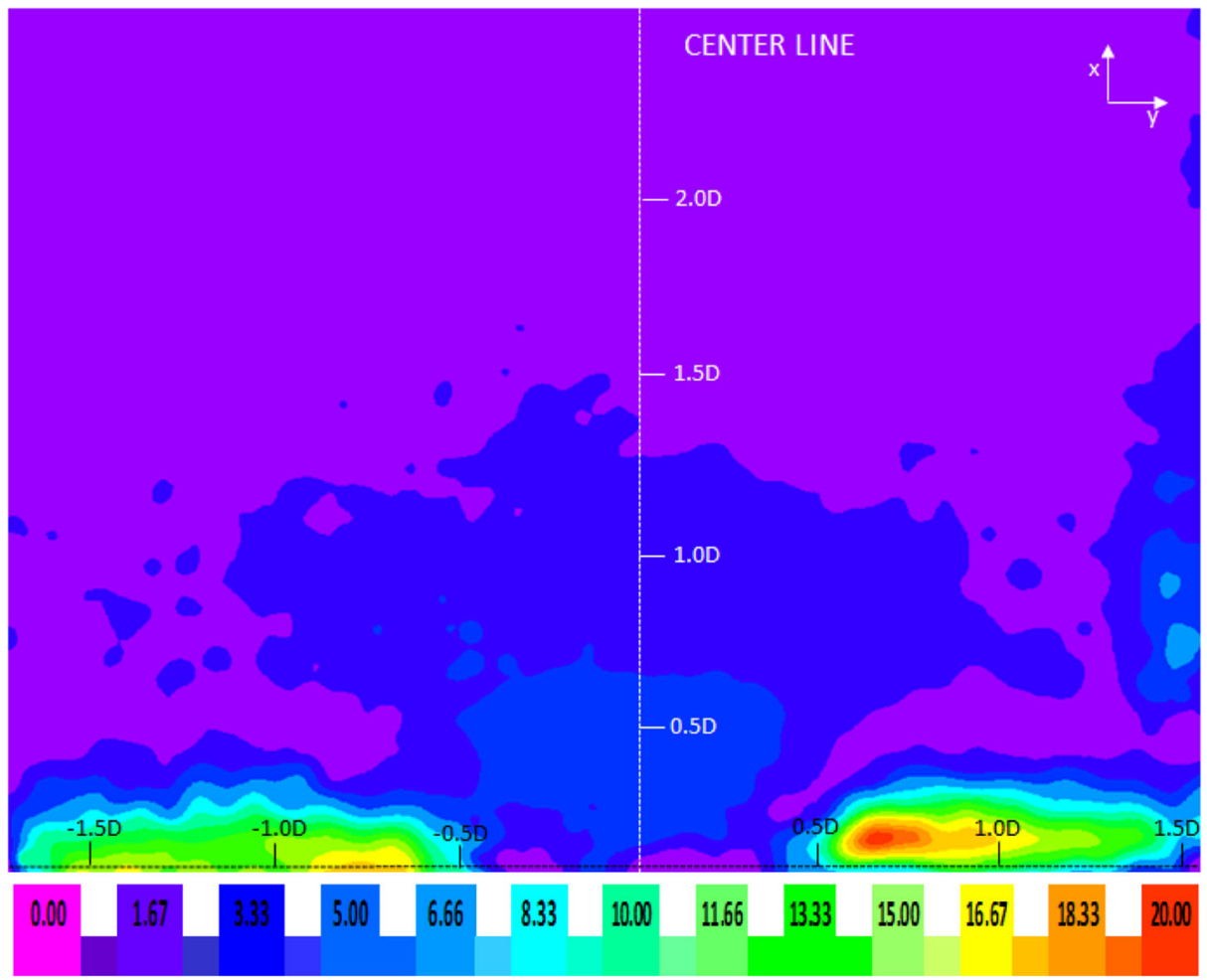

Fig. 6 CoJF shape. $\operatorname{Re}^{\sim} 15,500,|\Delta X| / D=0.000$, equivalence ratio $0.58, \alpha=45^{\circ}$. Total velocity in $[\mathrm{m} / \mathrm{s}]$
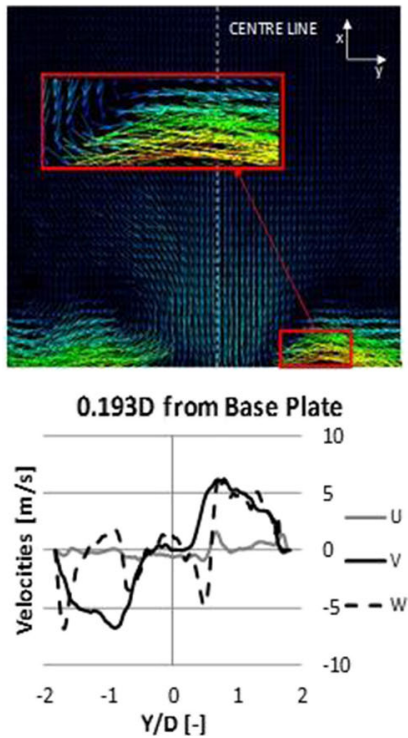

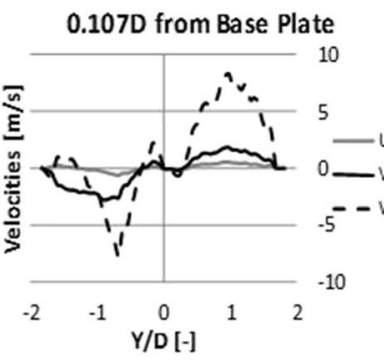

$0.236 \mathrm{D}$ from Base Plate

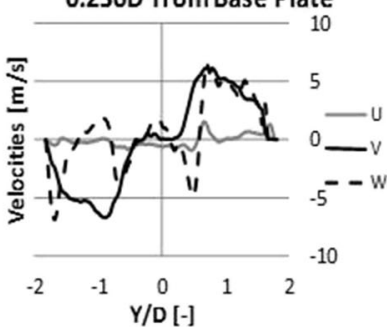

0.150D from Base Plate

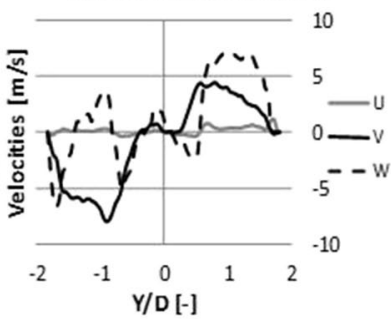

$0.279 \mathrm{D}$ from Base Plate

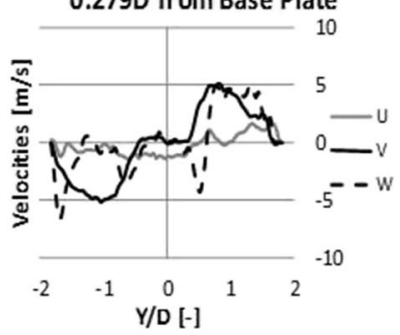

Fig. 7 CoJF shape. $\operatorname{Re}^{\sim} 15,500,|\Delta \mathrm{X}| / \mathrm{D}=0.000$, equivalence ratio $0.58, \alpha=45^{\circ}$ 

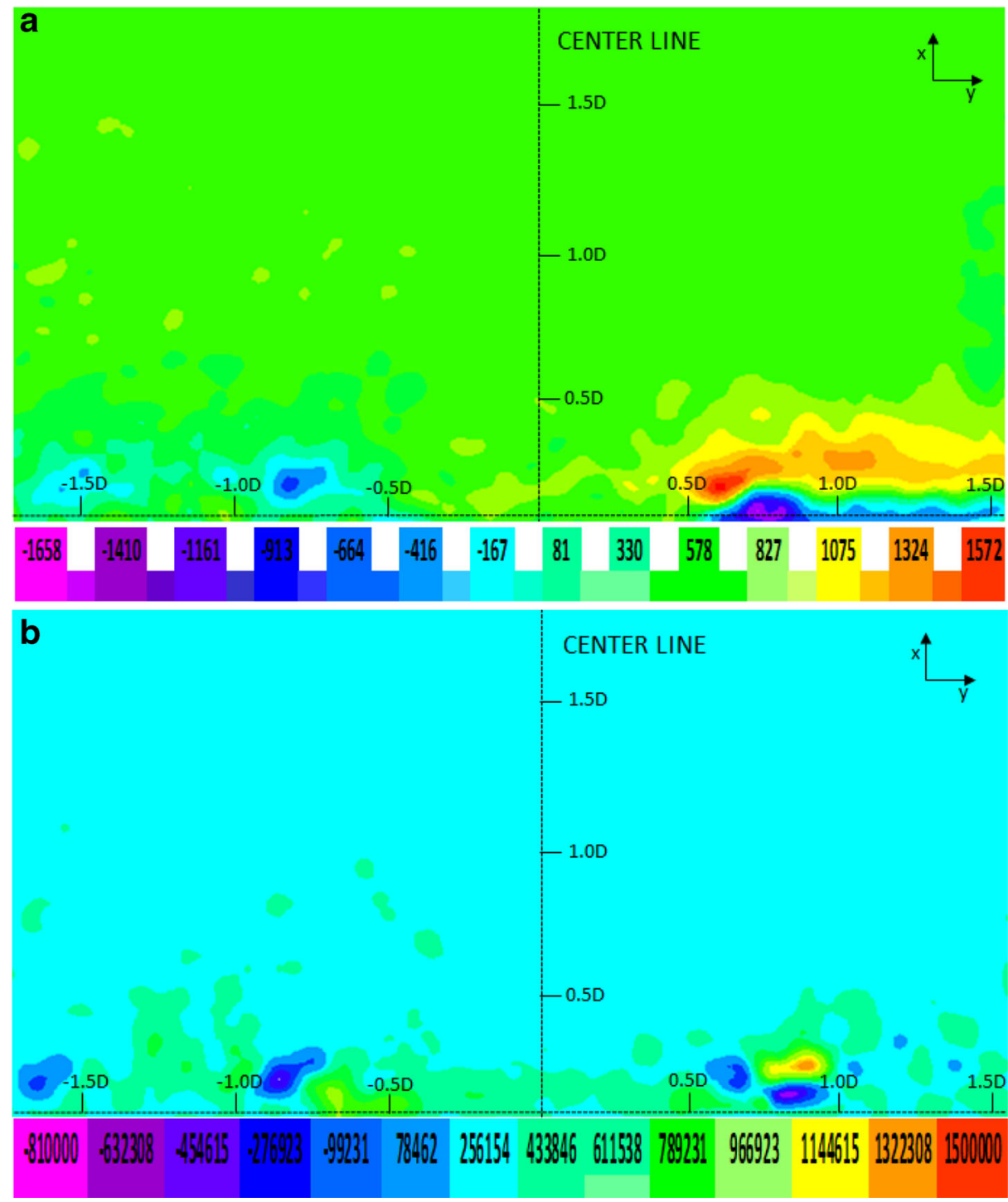

Fig. 8 CoJ. a) Vorticity analysis. Units in $[1 / \mathrm{s}]$. b) Swirling strength. Units in $\left[\mathrm{m} / \mathrm{s}^{2}\right]$

different Re and all experiments showed that it was at this $|\Delta \mathrm{X}| / \mathrm{D}$ when the system changed suddenly from a conical OJF-MS to a CoJF flow and vice versa. It was expected that the transition would go only in one direction instead of being reversible. Discussion on this point will follow in the next section.

High Speed Photography experiments showed the transition of the trapped vortex and how this is destroyed when the flow recovers its OJF-MS pattern, Figs. 9 and 10. First moments of the transition show an attached flow that is bending at a constant frequency as a product of 


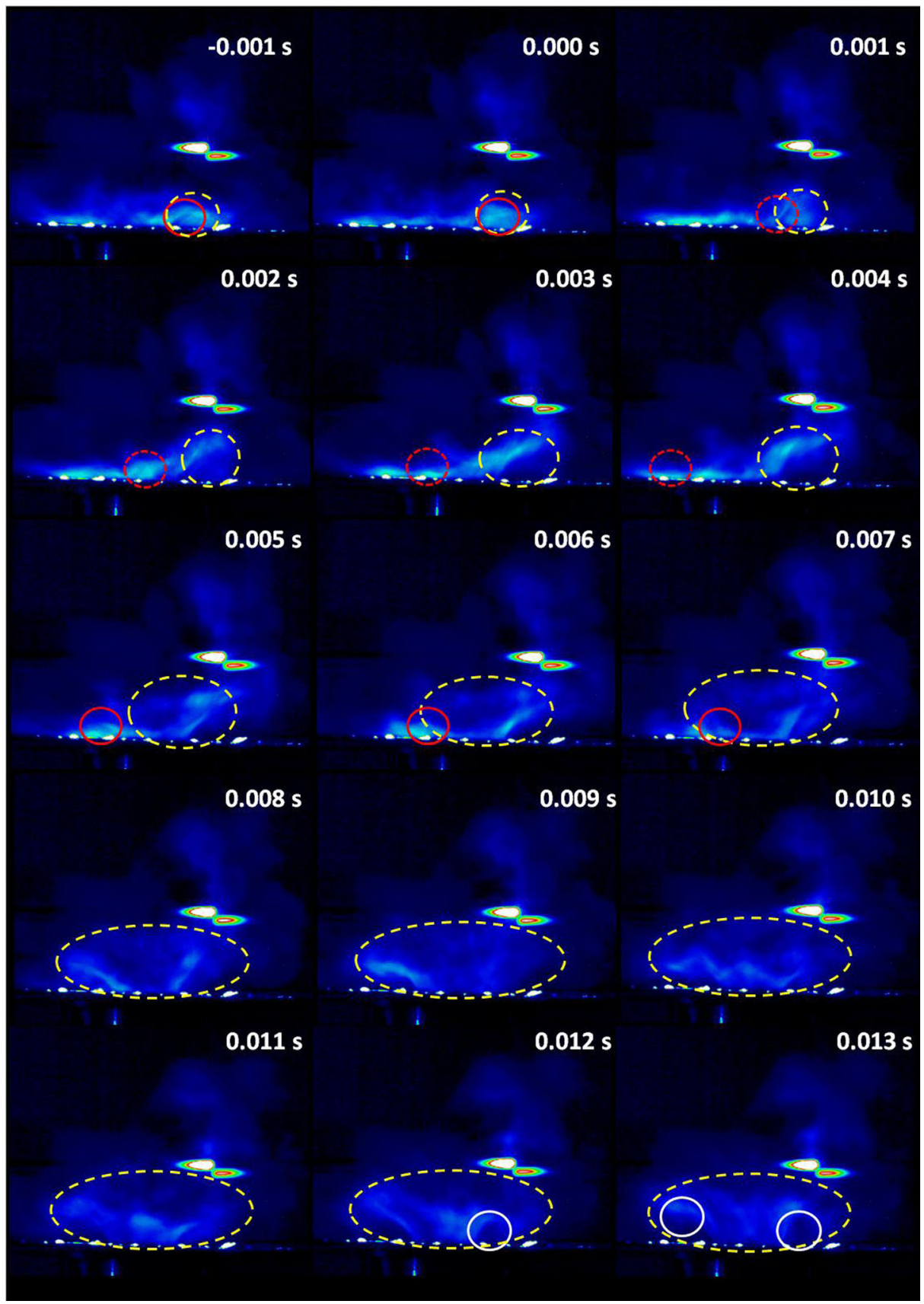

Fig. 9 High Speed Photography during transition, $\operatorname{Re}^{\sim} 10,100$ and $|\Delta X| / D=0.082$. Detached flame (yellow dotted line), Trapped Vortex (red line; in front of the flame - continuous line; behind the flame - dotted line) and PVC (white line) can be observed at different times. Flame lifting is slower than TV movement around the flame 
the trapped vortex. When the system loses its stability, the vortex is unable to keep the flame attached, $\mathrm{t}=0.000 \mathrm{~s}$. The flame starts lifting whilst the trapped vortex moves around the burner from $t=0.001 \mathrm{~s}$. Figure 9 at $\mathrm{t}=0.001 \mathrm{~s}$ shows the movement of the TV behind the flame. As the trapped vortex keeps moving around the nozzle, the flame detaches causing series of waves across the profile from $0.001 \mathrm{~s}<\mathrm{t}<0.007 \mathrm{~s}$, Fig. 10a. The TV seems to be responsible for the detachment. At $\mathrm{t}=0.007 \mathrm{~s}$ the $\mathrm{TV}$ is in front of the flame, directly facing the high speed camera, after completing a cycle around the nozzle. The trapped vortex disappears when it reaches the first point of detachment of the flame at $t=0.008 \mathrm{~s}$. A moment of extreme chaotic behaviour follows from $t=0.009 \mathrm{~s}$, with an undefined shape of the flame at $t=0.010 \mathrm{~s}$, Fig. 10b. Suddenly, a PVC appears opposite to the place where the trapped vortex would be if it was still present in the field, $t=0.012 \mathrm{~s}$, grapping the flame from that moment onwards, Fig. 10c. From these results it seems that the trapped vortex has progressed into an External Recirculation Zone, with the TV being completely independent towards the appearance of the PVC. The remnants of the vortices across the flame that appear during the chaotic behaviour might start to gather in order to form the PVC.

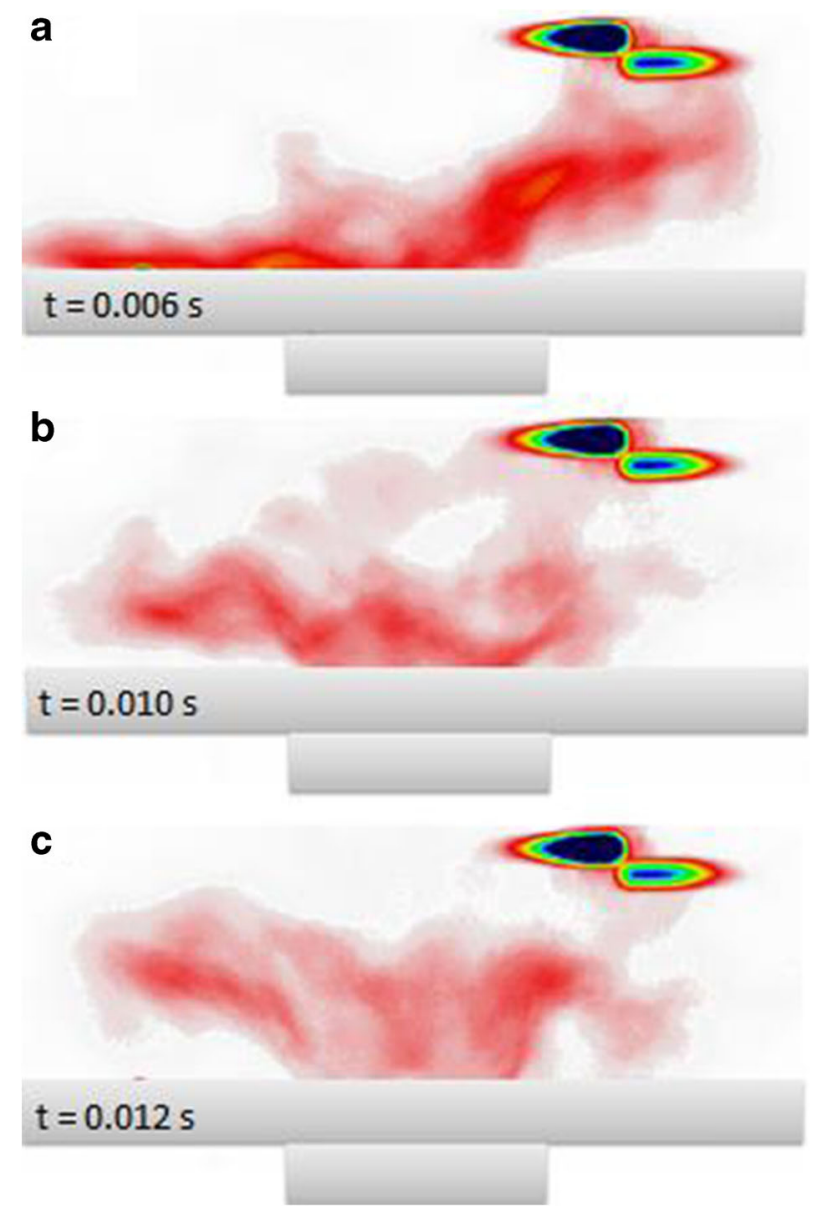

Fig. 10 High Speed Photography during transition, details of 3 stages using inverted colouring. a) Trapped Vortex moving around nozzle; b) Chaotic behaviour; c) PVC around the flame 


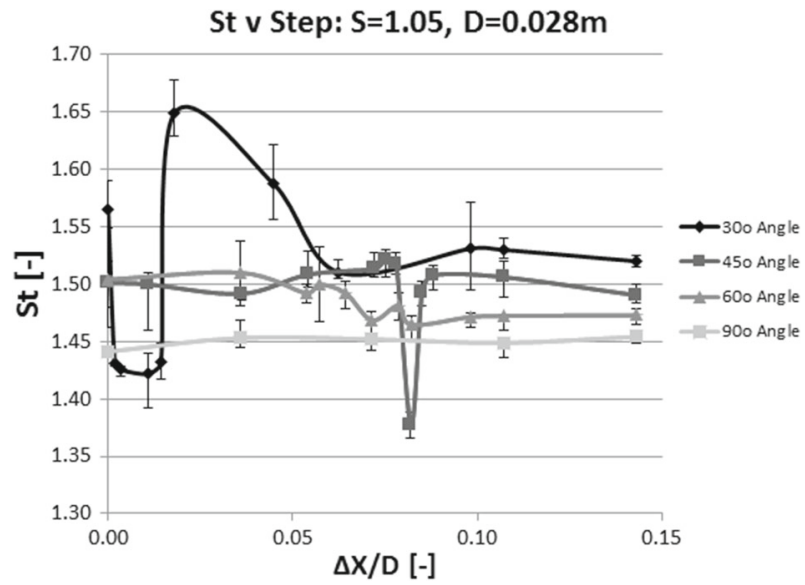

Fig. 11 Comparison between different geometries. St $v|\Delta X| / D$. Abrupt and longer transition for geometries close to $\alpha={ }^{\circ} 0$. Greater deviation also is obtained from small $\alpha$ profiles

\subsection{Isothermal experiments}

Isothermal experiments were carried out and similar patterns to the ones detected during combustion were observed. Although the decrease in density that occurred as the consequence of higher temperature affected the swirl number [1], it was noticed that the same transition happened at $|\Delta \mathrm{X}| / \mathrm{D}=0.082$ with $\alpha=45^{\circ}$ using the same Re numbers. This phenomenon of superimposition was previously observed by Vanoverberghe [10,31]. As the high temperatures of the Coanda flame posed a threat to the Stereo PIV cameras and the flowrates were going to be kept at the same values, it was decided to shift experiments to isothermal conditions.

Different nozzles were used with various $\alpha$. The frequencies of the fluctuating precessional signal were monitored, the data being plotted as Strouhal number. Comparison between all the cases was performed against the step size, Fig. 11 . At $\alpha=30^{\circ}$, the burner experiences a very abrupt reduction of frequency during transition that last for several $|\Delta \mathrm{X}| / \mathrm{D}$ steps. Before recovering stability at $1.52 \mathrm{St}$, there is a considerable jump in frequency at $|\Delta \mathrm{X}| / \mathrm{D}=0.018$. Although this big jump in not fully understood yet, the decay seems to be linked to the strength of the structures during transition. A similar effect happens at $\alpha=45^{\circ}$, being first stable with a CoJ pattern that is followed by an OJF-MS. However, the transition is not as abrupt and the recovery is faster. When the geometry is changed to $\alpha=60^{\circ}$ there is a minimal change in frequency that is barely noticeable. Finally, $\alpha=90^{\circ}$ does not show any change in frequency since the flow never experiences any transition to CoJ, always staying as an OJF-MS.

There is some scatter in the results with Strouhal number variations up to 0.077 , with typical Strouhal numbers being around 1.52 and 1.45, depending on the geometry. The only exceptions are for the $|\Delta \mathrm{X}| / \mathrm{D}$ where transition occurs. As the TV-ERZ structure radically changes between the two flow states there is a frequency change up to $20-30 \mathrm{~Hz}$. The phenomenon seems like a rupture point where the system losses its stability, regaining it by decreasing the St number. Thus, the geometry creates different transitions going from abrupt to very smooth as the geometry is closer to $\alpha=90^{\circ}$. A longer transition occurs at $\alpha=30^{\circ}$, whilst at $45^{\circ}$ and $60^{\circ}$ the phenomenon occurs at a similar step size of $|\Delta \mathrm{X}| / \mathrm{D}=0.082$ with a quick progression to stability. It is clear that once that stability is regained, the OJF-MS flow has a lower frequency than the CoJF. 

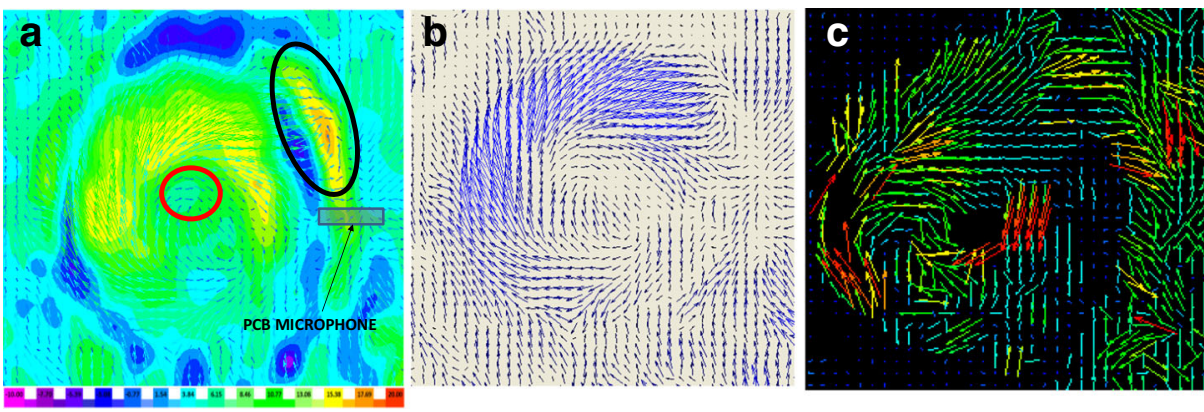

Fig. 12 OJF-MS a) axial velocity contours; b) Phase averaged radial-tangential velocity vectors exactly at the outlet of the nozzle; c) Instantaneous radial-tangential velocity vectors exactly at the outlet of the nozzle. $\operatorname{Re}^{\sim} 19,000, \Delta \mathrm{Y} / \mathrm{D}=0.082$ and $\alpha=45^{\circ} . \mathrm{PVC}($ red $)$ and ERZ (black) encircled. Velocity in $[\mathrm{m} / \mathrm{s}]$

Radial-Tangential velocity measurements were carried out to complement the experimental study. The results showed the transient and complex nature of both the TV and ERZ, Figs. 12 and 13. Standard deviations for both cameras were $\sim 6.8 \mathrm{~m} / \mathrm{s}$ with a mean value $\sim 10 \mathrm{~m} / \mathrm{s}$, which shows the highly complex and turbulent system that has appeared in the vicinity of the structures. It is clear that on both states a very strong TV or ERZ has been formed. This matches the axial-radial maps, with the strong signal arising from the highest velocity peak produced by the structures. The negative values denote a flow that is moving towards the exhaust plate, whilst the positive velocities emphasize the strong outgoing flow produced by the rotation of these bodies.

\section{Discussion}

An important point arising from the OJF-MS case is that the frequency comes from the PVC/HMFR structure. However, the PVC and HMFR have been suppressed in the CoJ as a consequence of the interaction between the TV and the shearing flow. Thus, the triggering signal for the phase locking arises from other structures rather than the PVC. In all the experiments, the CoJF has a St number slightly higher than the OJF-MS, confirming that the
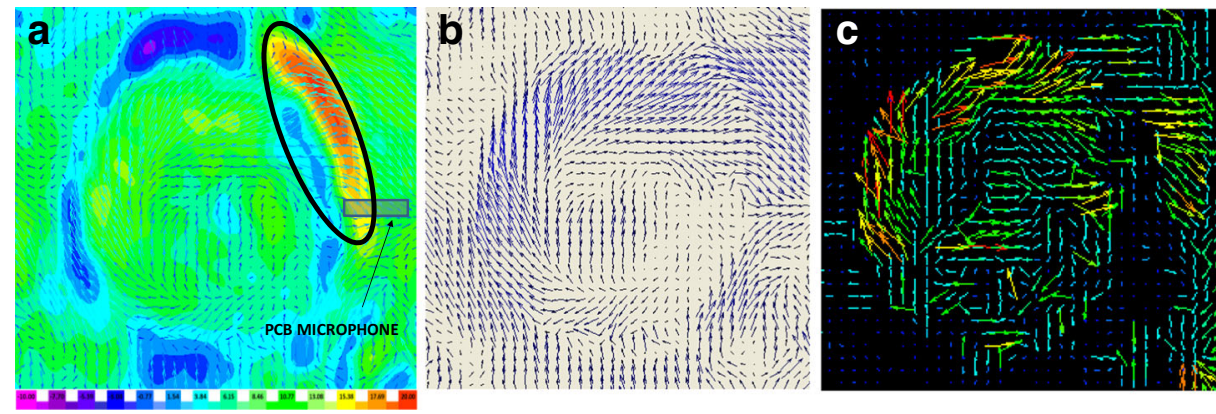

Fig. $13 \mathrm{CoJ}$ a) axial velocity contours; b) Phase averaged radial-tangential velocity vectors exactly at the outlet of the nozzle; c) Instantaneous radial-tangential velocity vectors exactly at the outlet of the nozzle. $\operatorname{Re}^{\sim} 19,000, \Delta \mathrm{Y} / \mathrm{D}=0.082$ and $\alpha=45^{\circ}$. TV (black) encircled. Velocity in $[\mathrm{m} / \mathrm{s}]$ 
structure that triggers the system is different and faster. Compared to the OJF-MS flow, the region has increased by around $150 \%$ of its original dimensions. Therefore, results allow to formulate the following theory,

A) Coanda stabilization: Initial moments of the CoJ show the high velocity flow leaving the exhaust nozzle creating an ERZ which pulls the jet onto the exhaust plate due to pressure difference, thus forming a TV whose strength attaches the flow to the plate. However, this stable TV is faster than other structures observed in open jet flows, as observed in Fig. 11. This structure as any other vortex will increase the stretch of the flame as it moves around the burner $[39,40]$. This extra air combined with the stretch of the flame will reduce local equivalence ratios. However, this is not a problem under these conditions as the strong vortex will keep the negative pressure where it is positioned to maintain the CoJ profile.

The only way that this trapped vortex can resist the attachment of the flow and the compression of the shearing flow is through a rise in strength, increasing its velocity. The trapped vortex augments the interaction with the surrounding flow, increasing recirculation and anchoring the flame, behaving in the same way as a Central Recirculation Zone. Therefore, the structure might be passing through a similar phenomenon of Vortex Breakdown as the one observed in the CRZ. Therefore, a Coanda Vortex Breakdown (COVB) might be taking place. This COVB, a stronger, faster Trapped Vortex, will increase the velocity and strength of the flow close to the flat plate through the change from supercritical to subcritical conditions, as observed in the stretch of the flow and further expansion downstream, Fig. 8 and 13. Experimental results show how the COVB pulls more resources from its surrounding increasing its velocity. This alters the flow in a similar way as a cavity profile will lead to the impingement of the shear layer in the opposite side of such a cavity [41, 42]. Nevertheless, the strong attachment of the COVB to the plate will ensure that the CoJ features remain unaltered.

B) Transition: During transition, the COVB loses its strength which leads to a reduction of its frequency, Fig. 11. Being capable only to sustain itself through this unstable conditions, the structure cannot move at the same speed, reducing St and evolving to a simpler TV. Extra air intake produced at the critical $|\Delta \mathrm{X}| / \mathrm{D}$ at which the system is in transition will reduce even more the instantaneous local equivalence ratio where the TV is located. Thus, wherever the TV has passed, the flame will be slower and unable to reattach as a CoJF making the transition to an OJF-MS. This transition happens with the destruction of the flow as a consequence of the appearance of a Central Vortex Breakdown. The chaotic patterns and formation of vortices across the flow allow the gathering of the latter around the newly formed CRZ, which provides the necessary energy for the formation of the PVC. Once that stability has been regained as an OJF-MS the structure reduces its suctioning characteristics, as observed from the PIV results, Fig. 12. Therefore, the frequency of the system is different and lower, as observed during the experiments. Reversibility can also be perceived as the OJF-MS will re-appear and the normal Coanda effect occurs. It is at this point where both effects are in balance, and very small perturbations will cause the transition from one state to the other.

The nozzle angle is another parameter that plays an important role in the transition of the flow. Fig. 11 shows how St changes and decreases with the increase of $\alpha$. Moreover, the abrupt transition observed at low angles is barely noticeable at $\alpha=60^{\circ}$. This phenomenon is thought to be caused by both the position and reduced strength of the TV. The greater $\alpha$ is, the greater the interaction of the TV with the axial flow will be. Thus, at low $\alpha$ the TV is partially protected by the geometry, being stabilized by the pressure differences and incoming air. However, there is a point when the shearing flow drags this partial section of the TV destabilising the 
entire structure. If the body is not strong enough to resist this convection, an OJF-MS will appear. A similar phenomenon was observed by Najm and Ghoniem [43] who reported the convection of recirculating flow regions in cavities. When $\alpha$ is increased, the shearing flow pushes this partial section of the TV out of its stability position sooner. However, the recovery of the CoJF happens as the pressure close to the nozzle is still low enough to pull back the flow towards the plate.

C) Open Jet stabilization: As soon as this process stops due to changes in geometry, density or sudden combustion, the flow will recover its OJF-MS features, allowing the appearance of the Central Vortex Breakdown with its CRZ and the appearance of the PVC, ERZ and HMFR. At larger step sizes, the flow is incapable of bending enough to avoid the incoming air to increase the pressure, thus ensuring that the TV will never appear.

\section{Conclusions}

Experiments were carried out to observe and understand the transition of a CoJF to/from a OJF-MS. Special emphasis was done on the progression of different coherent structures across the profile. It was found that the process occurs at a particular geometry and step size, with a shift in frequency produced by the leading structure due to its strength and air entrainment. Stability of the CoJ occurs after a Coanda Vortex Breakdown has occurred, a process similar to the one observed in the central region of the flow under an OJF-MS. This stronger Trapped Vortex will be the principal structure in the system, contrary to the OJF-MS system where the CRZ/PVC/HMFR are the leading structures. Results allowed formulation of the following theory; as the step size is increased, a COVB will evolve into a slower Trapped Vortex. The stability of this Trapped Vortex will depend on its position in the field relative to the shearing flow, with a protected vortex being more difficult to convect downstream. This TV will experience a decay in frequency as a consequence of having just momentum to stabilize itself, reducing its impinging effect on the opposite and surrounding flows. During transition, the Trapped Vortex will still be present and its movement around the nozzle will finish the lift of the flame due to both local stretch and reduction of equivalence ratio. Once the TV reaches the first point of detachment it will be dragged by the shearing flow forming an External Recirculation Zone. This process will occur with a very chaotic behavior consequence of the appearance of the Central Vortex Breakdown, leading to the destruction of any coherent pattern and vortical structures in the system. The remaining vortices, located all around the field, will start to gather due to the presence of the newly formed Central Recirculation Zone, creating a structure named Precessing Vortex Core. Temporal-resolution studies are required to confirm this theory and to provide complete characterization of the COVB.

Acknowledgments Mr. Hesham Baej gratefully acknowledges the support of the Libyan Embassy and the Libyan Cultural and Education Bureau in London. The authors would like to thank Dr. Elisabetta de Angelis for her contributions reviewing the content of this paper. The authors would also like to thank the invaluable inputs from the reviewers. Their comments and suggestions have considerably improved the quality of this work.

Open Access This article is distributed under the terms of the Creative Commons Attribution 4.0 International License (http://creativecommons.org/licenses/by/4.0/), which permits unrestricted use, distribution, and reproduction in any medium, provided you give appropriate credit to the original author(s) and the source, provide a link to the Creative Commons license, and indicate if changes were made. 


\section{References}

1. Syred, N.: A review of oscillation mechanisms and the role of the precessing vortex core (PVC) in swirl combustion systems. Prog. Energy Combust. Sci. 32, 93-161 (2006)

2. Syred, N., Beer, J.M.: Combustion in swirling flows: a review. Combust. Flame 23, 143-201 (1974)

3. Gupta, A.K., Lilley, D.J., Syred, N.: Swirl flows, Abacus Press, Tunbridge Wells UK (1984)

4. Lieuwen, T., Yang, V.: Combustion Instabilities in Gas Turbine Engines. In: Progress in Astronautics Aeronautics, AIAA U.S.A, vol. 210, pp. 213-276. AIAA, U.S.A. (2005)

5. Dawson, J.R., Rodriquez-Martinez, V.M., Syred, N., O'Doherty, T.: Low Frequency Combustion Oscillations in a Swirl Burner Furnace, AIAA Int Meet Paper 2004-9197. Nevada, U.S.A (2004)

6. Dawson, J.R., Rodriquez-Martinez, V.M., Syred, N., O'Doherty, T.: The effect of combustion instability on the structure of recirculation zones in con fined swirling flames. Comput. Sci. Technol. 177, 1-22 (2005)

7. Vanierschot, M., Van den Bulck, E.: Hysteresis in flow patterns in annular swirling jets. Exp. Thermal Fluid Sci. 31(6), 513-524 (2007)

8. Lubert, C.: On Some Recent Applications of the Coanda Effect to Acoustics, Proc Meet Acoust 11, ref $040006(2011)$

9. Osamor, F.A., Ahlert, R.C.: Oil and water separation, state of the art, EPA, Industrial Environmental Research Laboratory EPA-600/2-78-069 (1978)

10. Vanoverberghe, K.: Flow, Turbulence and Combustion of Premixed Swirling Jet Flames. PhD Thesis, Katholieke Universiteit Leuven, Leuven, Belgium (2004)

11. Sidhu, B.S., Syred, N., Styles, A.C.: Flow and General Characteristics of High Performance Diodes, ASME Winter Meet, pp. 113-122. U.S.A, Chicago (1980)

12. Lubert, C., Shafer, R.: Shock Associated Noise Generation in Curved Turbulent Coanda Wall Jets, Proc Meet Acoust 14, ref 040002 (2011)

13. Wing, D.J.: Static investigation of two fluidic Thrust-Vectoring concepts on a Two-Dimensional Convergent-Divergent nozzle, NASA. Tech Memo, 4574 (1994)

14. Kniesburges, S., Hesselmann, C., Becker, S., Schlücker, E., Döllinger, M.: Influence of vortical flow structures on the glottal jet location in the supraglottal region. J. Voice 27(5), 531-544 (2013)

15. Lubert, C.: Application of turbulent mixing noise thoery to flows over coanda surfaces. Int. J. Acoust. Vib. 13(1), 17-30 (2008)

16. Aleseenko, S., Kuibin, P., Okulov, V., Shtork, S.: Helical vortex in swirl flow. J. Fluid Mech. 382, 195-243 (1999)

17. Mirkov, N., Rasuo, B.: Maneuverability of an UAV with Coanda Effect Based Lift Production, 28th Int Cong Aeronaut Sci (ICAS), pp. 1-6 U.S.A (2012)

18. Rumsey, C.L., Nishino, T.: Numerical Study Comparing RANS and LES Approaches on a Circulation Control Airfoil, 49Th AIAA Sci Meet, ref. AIAA 2011-1179. U.S.A, Orlando, Florida (2011)

19. Dragan, V., Stanciu, V.: Contribution regarding a fluid barrier super circulation technique. UPB Scie. Bull Series D Mech. Engin. 75(2), 17-30 (2013)

20. Tavakoli, E., Hosseini, R.: Large eddy simulation of turbulent flow and mass transfer in far field of swirl diffusers. Energy Build. 59, 194-202 (2013)

21. Singh, N.K., Ramamurthi, K.: Formation of coanda jet from Sharp-Edged swirl nozzle with base plate. Exp. Thermal Fluid Sci. 33, 675-682 (2009)

22. Nishino, T., Sharif, K.: Effect of jet Nozzle-Lip momentum loss on circulation control airfoil performance. AIAA J. 50(3), 551-558 (2012)

23. Turrell, M.D., Stopford, P.J., Syed, K., Buchanan, E.: CFD Simulations of the flow within and downstream of high swirl lean premixed gas turbine combustors. Proc. ASME 1, 31-38 (2004)

24. Jakirlic, S., Hanjalic, K., Tropea, C.: Modelling rotating and swirling turbulent flows, a perpetual challenge. AIAA J. 40(10), 1984-1997 (2002)

25. Sadiki, A., Maltseva, A., Wegnera, B., Flemminga, F., Kempfa, A., Janickaa, J.: Unsteady Methods (URANS and LES) for simulation of combustion systems. Int. J. Thermal Sci. 45(8), 760-773 (2006)

26. Jochmann, P.: Numerical simulation of a precessing vortex breakdown. Int. J. Heat Fluid Flow 27, 192203 (2006)

27. Lucca-Negro, O., O’Doherty, T.: Vortex Breakdown: a review. Prog. Energy Combust. Sci. 27, 431-481 (2001)

28. Candel, S., Durox, D., Schuller, T., Bourgouin, J.F., Moeck, J.: Progress in swirling flames and annular combustor dynamics, European Combust Meet, pp. 1-11 Sweden (2013) 
29. Candel, S., Durox, D., Schuller, T., Bourgouin, J.F., Moeck, J.: Dynamics of swirling flames. Annual Review of Fluid Mech. 46, 147-173 (2014)

30. Mullyadzhanov, R., Hadziabdic, M., Hanjalic, K.: LES Investigation of the hysteresis regime in the cold model of a Rotating-Pipe swirl burner, flow. Turbulence Combust. 94, 175-198 (2015)

31. Vanierschot, M., Van den Bulck, E.: Numerical study of hysteresis in annular swirling jets with a steppedconical nozzle. Int. J. Numerical Methods Fluids 54, 313-324 (2007)

32. Vanierschot, M., Van den Bulck, E.: Computation of a drastic flow pattern change in an annular swirling jet caused by a small change in inlet swirl. Int. J. Numerical Methods Fluids 59(5), 577-592 (2009)

33. Abdulsada, M., Syred, N., Bowen, P., O’Doherty, T., Griffiths, A., Marsh, R., Crayford, A.: Effect of exhaust confinement and fuel type upon the blowoff limits and fuel switching ability of swirl combustors. App. Thermal Eng. 48, 426-435 (2012)

34. Vigueras-Zuniga, M.O., Valera-Medina, A., Syred, N.: Studies of the precessing vortex core in swirling flows. J. App. Res. Tech. 10(3), 755-765 (2012)

35. Valera-Medina, A., Syred, N., Griffiths, A.: Visualization of coherent structures in a swirl burner under isothermal conditions. Combust. Flame 159, 1723-1734 (2009)

36. Zhou, J., Adrian, R.J., Balachandar, S., Kendall, T.M.: Mechanisms for generation of coherent packets of hairpin vortices in channel flow. J. Fluid Mech. 387, 353-359 (1999)

37. Adrian, R.J., Christensen, K.T., Liu, Z.C.: Analysis and interpretation of instantaneous turbulent velocity fields. Exp. Fluids 29, 275-290 (2000)

38. Dantec Dynamics: DynamicStudio Documentation Swirling Strenght Script (2009) [online] http://www. dantecdynamics.com/, [Accessed 12 ${ }^{\text {th }}$ Dec 2015]

39. Bomminayuni, S., Stoesser, T.: Turbulence statistics in an Open-Channel flow over a rough bed. J. Hydraul Eng. 137(11), 1347-1358 (2011)

40. Lieuwen, T.: Unsteady combustor physics, cambridge university press. USA, 404 (2012)

41. Stohr, S., Arndt, C.M., Meier, W.: Effects of Damköhler number on vortex flame interaction in gas turbine model combustor. Proc. Combust. Inst. 34, 3107-3115 (2013)

42. Rockwell, D.: Oscillations of impinging shear layers. AIAA J. 21(5), 645-664 (1983)

43. Najm, H.N., Ghoniem, A.F.: A numerical simulation of the convective instability in a dump combustor. AIAA J. 29, 911-919 (1991) 